\title{
Electrochemical Immunosensor for Carcinoembryonic Antigen Based on Antigen Immobilization in Gold Nanoparticles Modified Chitosan Membrane
}

\author{
Jiehua LiN, Wei Qu, and Shusheng ZHANG ${ }^{\dagger}$ \\ College of Chemistry and Molecular Engineering, Qingdao University of Science \& Technology, \\ Qingdao 266042, P. R. China
}

\begin{abstract}
A disposable electrochemical immunosensor for carcinoembryonic antigen (CEA) was proposed based on the antigen immobilized in a colloidal gold nanoparticles modified chitosan membrane on the surface of an indium-tin oxide (ITO) electrode. The different membranes were characterized by scanning electron microscope and electrochemical methods. Based on a competitive immunoassay format, the immobilized antigen of the immunosensor was incubated with a horseradish peroxidase (HRP) labeled antibody and sample CEA antigen, and the formed immunoconjugate in the immunosensor was detected by an $o$-phenylenediamine- $\mathrm{H}_{2} \mathrm{O}_{2}-\mathrm{HRP}$ electrochemical system. Under the optimal experimental conditions, the electrocatalytic current decreased linearly with the competitive mechanism. CEA could be determined in the linear range from 2.0 to $20 \mathrm{ng} / \mathrm{ml}$ with a detection limit of $1.0 \mathrm{ng} / \mathrm{ml}$. The prepared CEA immunosensor is not only economic due to the low-cost ITO electrode obtained from industrial mass production, but is also capable with good stability and reproducibility for batch fabrication.
\end{abstract}

(Received February 28, 2007; Accepted April 4, 2007; Published September 10, 2007)

\section{Introduction}

Carcinoembryonic antigen (CEA), with the characteristic of human embryo antigen, is an acidic glycoprotein with a molecular weight of about $200 \mathrm{kDa}$. At first, it was described as a specific indicator of colorectal carcinoma. Lately it was found that CEA has been expressed in many malignancies. ${ }^{1,2}$ The concentration of serum CEA is related to colon cancer, ${ }^{3}$ lung cancer, ${ }^{4,5}$ ovarian carcinoma, ${ }^{6}$ breast cancer, ${ }^{7-9}$ cystadenocarcinoma ${ }^{10}$ and others. ${ }^{11-14}$ Because the CEA level in serum is also related to the state of a tumor, it can be directly used for the evaluating curative effect, judging recrudescence or metastasis as a marker. ${ }^{15}$ Thus, detection of the serum CEA level plays an important role in an initial diagnostic evaluation and any follow-up examination during therapy. A number of immunoassay methods to detect CEA levels in serum have been reported, such as chemiluminescence immunoassay, ${ }^{16-18}$ electrochemical immunoassay, ${ }^{15}$ enzyme-linked immunosorbent assay, ${ }^{19,20}$ fluoroimmunoassay, ${ }^{21}$ liposome immunoassay ${ }^{22}$ and radioimmunoassay. ${ }^{23}$

An indium-tin oxide (ITO) electrode exhibits prominent characteristics, such as excellent optical transparency, high electrical conductivity, a wide electrochemical working window, excellent substrate adhesion, and stable electrochemical and physical properties. ${ }^{24-27}$ In addition, ITO film has been widely used in industrial applications, and therefore the ITO electrode could be available at very low cost due to mass production. Numerous studies have been conducted for the development and application of ITO electrodes on electronic and optical sensors, ${ }^{28}$ the direct electron transfer of proteins, ${ }^{29}$ electrochemical

$\doteqdot$ To whom correspondence should be addressed.

E-mail: shushzhang@126.com measurements, ${ }^{26,30}$ electrochemical nucleic acid biosensors ${ }^{31}$ and microfluidic on-chip devices. ${ }^{27,32,33}$ Usually, silane reagents have been attached to an activated ITO surface under wellcontrolled conditions for the immobilization of immunoreagents in the previous studies. ${ }^{34,35}$

During the past decades, plenty of natural polymers have been used as an immobilization matrix to prepare biosensors. Chitosan, an attractive natural biopolymer with the presence of reactive amino and hydroxyl functional groups, is one of the most promising type immobilization matrix, due to its desirable properties, including excellent membrane-forming ability, good adhesion, low cost, nontoxicity, high mechanical strength and hydrophilicity. ${ }^{36}$ It has been extensively used for immunoassay due to the presence of reactive amino and hydroxyl functional groups. ${ }^{37,38}$ Since the gold surface permits an easy absorption of protein molecules, colloidal gold nanoparticles have been used as the matrix for the immobilization of antibodies or antigens ${ }^{39-41}$ in order to retain the bioactivity of macromolecules. It was reported that 3-mercaptopropyl trimethoxysilane has been used to firmly adsorb gold colloids onto functionalized ITO surfaces in the previous studies. ${ }^{25,42,43}$ Huang and Yang explored an efficient method for the adsorption of colloidal gold nanoparticles on a chitosan membrane on a modified glassy carbon electrode. ${ }^{44}$ It was said that this absorption could provide an assembly of gold nanoparticle multilayers and a suitable microenvironment similar to the native environment of bioconjugates in electrochemical immunoassay. ${ }^{37}$ Thus, herein we chose this colloidal gold nanoparticles modified chitosan membrane (Au-chitosan) to immobilize CEA antigen. Although some studies using Au-chitosan to immobilize antigens on glassy carbon electrode and carbon-paste electrode have been reported, ${ }^{37,45}$ an optically transparent and conducting ITO electrode has never been used for the immobilization of antigen 
(a)

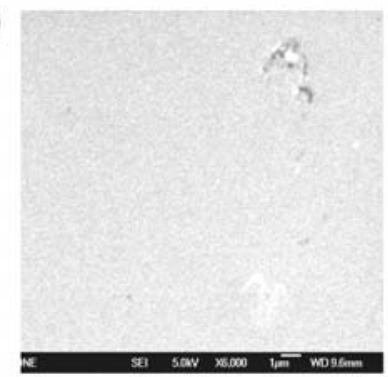

(b)

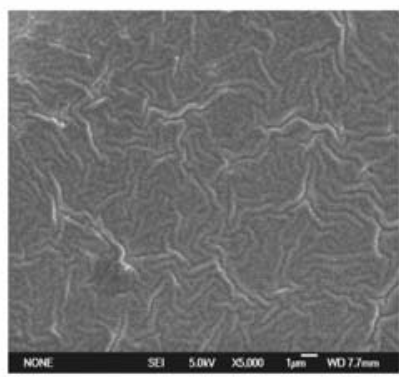

(c)

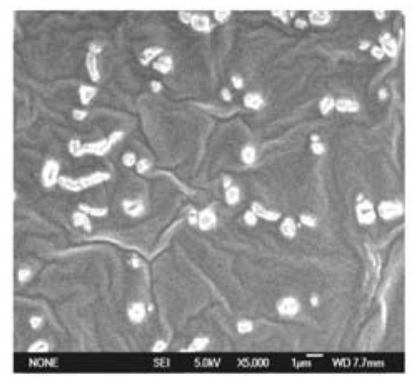

Fig. 1 Scanning electron micrographs of ITO electrodes coated with (a) a bare ITO electrode surface, (b) Au-chitosan and (c) CEA/Au-chitosan membranes.

to fabricate an electrochemical immunosensor. In our work, a disposable electrochemical immunosensor for the determination of CEA has been proposed based on the antigens immobilized on a Au-chitosan modified ITO electrode surface with the competitive immunoassay format. Compared with the results obtained by an immunoradiometric assay (IRMA), the developed immunosensor showed an acceptable accuracy. This strategy could be further developed for practical clinical detections of CEA and other important tumor markers.

\section{Experimental}

\section{Reagents and chemicals}

ITO sheets (resistance $100 \Omega /$ sqr) were purchased from Shenzhen YH Co. (China). CEA ELISA kits were purchased from Shanghai Jiemen Bio-tech Co. (China). The ELISA kits consisted of a series of CEA standard solutions from 0 to 120 $\mathrm{ng} / \mathrm{ml}$ concentration and a stock solution of HRP-labeled CEA antibody. Bovine serum albumin (BSA) was purchased from Sigma Chemical Company (St. Louis, MO, USA). Chitosan $\left(M_{\mathrm{W}} 1.9-3.1 \times 10^{5} ; 85-90 \%\right.$ deacetylation, Aldrich), $o$ phenylenediamine (OPD, Shanghai Biochemical Reagent Company, China), $\mathrm{H}_{2} \mathrm{O}_{2}$ (Shanghai Chemical Plant, China) were of analytical grade, and used as received. A chitosan solution (1 wt\%) was prepared by ultrasonically dissolving chitosan powder in $1 \%$ acetic acid. $\mathrm{A}_{2} \mathrm{O}_{2}$ working solution was prepared by appropriate dilution of a $30 \%$ solution in water daily. A $0.1 \mathrm{M}$ phosphate buffer solution (PBS) was used as an electrolyte in the measuring system; 0.01 M PBS was used to dilute the immunological reagents. Doubly distilled water was used throughout the experiment. All electrochemical reactions were carried out under an atmosphere of nitrogen.

\section{Apparatus}

Electrochemical measurements were performed on a $\mathrm{CHI}$ 832B electrochemical analyzer (Shanghai Chenhua) with a conventional three-electrode system comprised of platinum wire as an auxiliary electrode, $\mathrm{Ag} / \mathrm{AgCl}$ electrode as a reference and CEA modified ITO electrode as a working electrode.

Transmission electron micrograph (TEM) images were recorded on a JEM-1200EX (JEOL, Japan) transmission electron microscope. Scanning electron microscopic (SEM) image membranes were obtained with a field-emission SEM instrument JSM-6700F (JEOL, Japan).

\section{Preparations of CEA immunosensor}

The preparation of gold colloid nanoparticles was according to a method reported previously. ${ }^{46,47}$ Also, a Au-chitosan membrane modified ITO electrode was prepared according to
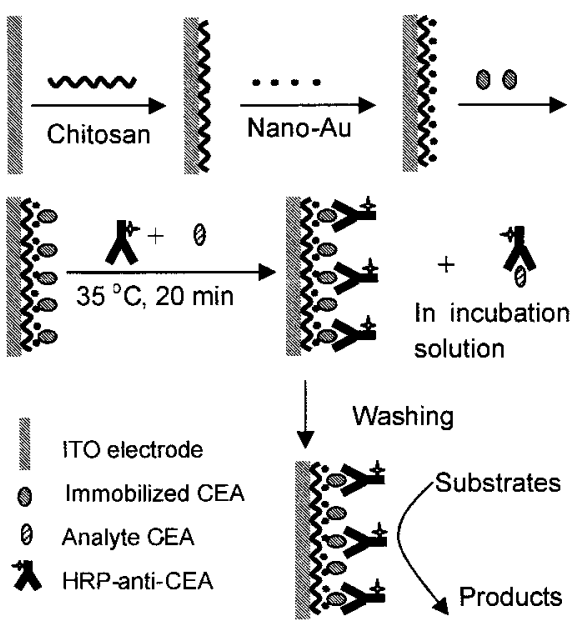

Fig. 2 Schematic diagram of the CEA/Au-chitosan/ITO immunosensor for determining the serum CEA level based on a competitive immunoassay.

references. ${ }^{34,47}$ The immobilization of CEA was accomplished by dripping $10 \mu \mathrm{l}$ of an $80 \mathrm{ng} / \mathrm{ml} \mathrm{CEA} \mathrm{standard} \mathrm{solution} \mathrm{onto}$ the above mentioned prepared membrane. After the evaporation of water at room temperature for about $5 \mathrm{~h}$, the electrode was rinsed thoroughly with double-distilled water, and then dipped in a BSA solution to block the active sites. After drying for $1 \mathrm{~h}$, the obtained immunosensor was kept at $4^{\circ} \mathrm{C}$ prior to use. The area of the working surface was about $5 \times 6 \mathrm{~mm}^{2}$. The immobilization was characterized by SEM, as shown in Fig. 1.

\section{Electrochemical competitive immunoassay for CEA}

The electrochemical detection of CEA was based on a competitive immunoassay format. A schematic diagram of the procedure is shown in Fig. 2. Firstly, a 5- $\mu$ l CEA standard or sample solution was mixed with $10 \mu \mathrm{l}$ of a diluted HRP labeled CEA antibody stock solution (diluted ratio, 1:1) to prepare the incubation solution. Then, the immobilized CEA antigen immunosensor was put in the incubation solution at $35^{\circ} \mathrm{C}$ for 20 min for the competitive immunoassay, and the immunoconjugate was formed on the surface of the immunosensor. After the reaction, the immunosensor was carefully rinsed with the doubly-distilled water. The electrochemical measurement was recorded in a 0.1 M PBS solution $(\mathrm{pH} 5.0)$ containing $2.0 \mathrm{mM}$ OPD and $0.5 \mathrm{mM} \mathrm{H}_{2} \mathrm{O}_{2}$, which was thoroughly deaerated with highly pure nitrogen for $15 \mathrm{~min}$ and maintained in a nitrogen atmosphere at $20 \pm 0.5^{\circ} \mathrm{C}$. Differential pulse voltammetric (DPV) measurements were performed from -300 to $-800 \mathrm{mV}$ 
with a pulse amplitude of $50 \mathrm{mV}$ and a pulse width of $50 \mathrm{~ms}$

\section{Results and Discussion}

Morphologies of different membranes on an ITO electrode surface The performance of an electrochemical immunosensor is related to its physical morphology. Thus, the surface morphology of the Au-chitosan membrane is a vital effect factor. The surface morphologies of the membranes were observed by SEM in this work. From the SEM image shown in Fig. 1, it can be seen that the Au-chitosan membrane showed a clean and homogenous network structure (Fig. 1b) compared to a bare ITO electrode (Fig. 1a). However, when a Au-chitosan membrane modified ITO electrode was dropped on CEA antigen, its SEM image showed aggregates of the trapped biomolecules with a regular distribution. Thus, the presence of the Au-chitosan membrane played an important role in the immobilization of CEA antigen on the ITO electrode surface (Fig. 1c).

\section{Electrochemical behaviors of modified ITO electrodes}

It is well known that HRP can catalyze the oxidation reaction of OPD by $\mathrm{H}_{2} \mathrm{O}_{2}$. The mechanism of enzymatic catalysis and the oxidation reaction has been described. ${ }^{48}$ No amperometric response of the Au-chitosan/ITO and CEA/Au-chitosan/ITO was observed in the absence of OPD and $\mathrm{H}_{2} \mathrm{O}_{2}$ in $0.1 \mathrm{M}$ PBS

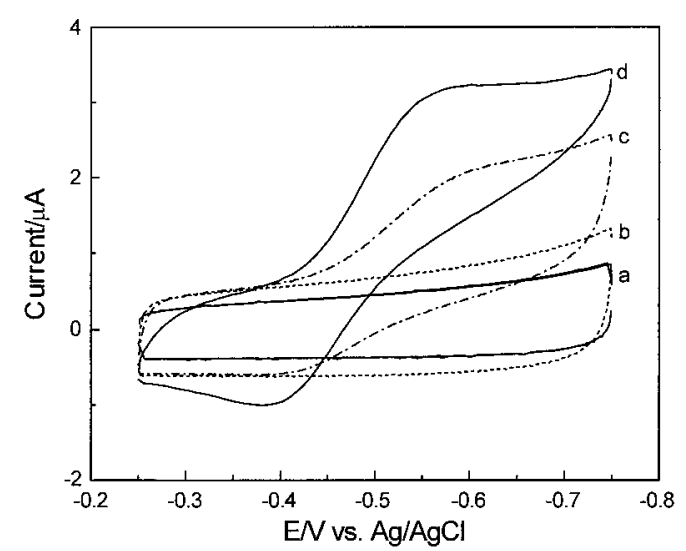

Fig. 3 Cyclic voltammograms of different electrodes at $50 \mathrm{mV} / \mathrm{s}$. (a) Au-chitosan/ITO, (b) CEA/Au-chitosan/ITO in $0.1 \mathrm{M} \mathrm{pH} 5.0$ PBS, (c) (b) $+2.0 \mathrm{mM} \mathrm{OPD}+0.25 \mathrm{mM} \mathrm{H}_{2} \mathrm{O}_{2}$, (d) HRP-antiCEA/CEA/Au-chitosan/ITO in $0.1 \mathrm{M} \mathrm{pH} 5.0 \mathrm{PBS}$ including $2.0 \mathrm{mM}$ $\mathrm{OPD}+0.5 \mathrm{mM} \mathrm{H}_{2} \mathrm{O}_{2}$.
(pH 5.0) (Figs. 3a and b). When $2.0 \mathrm{mM}$ OPD and $0.25 \mathrm{mM}$ $\mathrm{H}_{2} \mathrm{O}_{2}$ were added into PBS, the cyclic voltammogram of CEA/Au-chitosan/ITO at $50 \mathrm{mV} / \mathrm{s}$ showed a couple of oxidation and reduction peaks for OPD at -595 and $-402 \mathrm{mV}$ (Fig. 3c). After incubation, immunoconjugates on the surface of a $\mathrm{CEA} / \mathrm{Au}$-chitosan/ITO electrode were formed, and the HRP labeled antibody bound to the immobilized CEA antigen, leading to an electrocatalytic process in the presence of $2.0 \mathrm{mM}$ OPD and $0.25 \mathrm{mM} \mathrm{H}_{2} \mathrm{O}_{2}$ in $0.1 \mathrm{M}$ PBS (pH 5.0), which is shown in Fig. 3d. It can be seen that the reduction peak current of HRP/Au-chitosan/ITO dramatically increased and the oxidation peak current decreased. The pair of oxidation and reduction peaks for OPD was at -553 and $-406 \mathrm{mV}$ at $50 \mathrm{mV} / \mathrm{s}$.

\section{Optimal conditions for electrochemical detection}

The analytical performance of the CEA immunosensor was related to the concentrations of OPD and $\mathrm{H}_{2} \mathrm{O}_{2}$ in the detection solution and the $\mathrm{pH}$ value of the substrate solution. When the OPD concentration increased in $\mathrm{pH}$ 5.0 PBS containing $0.5 \mathrm{mM}$ $\mathrm{H}_{2} \mathrm{O}_{2}$, the DPV current response increased correspondingly, and reached the maximum value at a concentration of $2.0 \mathrm{mM}$ (Fig. 4a). Thus this concentration was chosen as the optimal OPD concentration. With increasing $\mathrm{H}_{2} \mathrm{O}_{2}$ concentration, the DPV current response of the obtained immunoconjugate increased and reached the maximum response in $\mathrm{pH}$ 5.0 PBS containing $2.0 \mathrm{mM}$ OPD at a $\mathrm{H}_{2} \mathrm{O}_{2}$ concentration of $0.5 \mathrm{mM}$, which could be used in the current work for the electrochemical competitive immunoassay (Fig. 4b). The $\mathrm{pH}$ value of the solution was also an important parameter and was examined in this work, as shown in Fig. 4c. The immunosensor after incubation with the analyte CEA and HRP-labeled anti-CEA in solution showed the maximum response at a $\mathrm{pH}$ value of 5.0 in $0.1 \mathrm{M}$ PBS containing $2.0 \mathrm{mM}$ OPD and $0.5 \mathrm{mM} \mathrm{H}_{2} \mathrm{O}_{2}$. Thus, $\mathrm{pH} 5.0$ was selected for the electrochemical immunoassay.

\section{Optimization of the immunoassay procedure}

The effect factors of the immunoassay procedure included the optimal dilution of HRP-labeled anti-CEA, the incubation temperature and the time for the immunoreaction. Further, the amount of CEA antigen immobilized on Au-chitosan/ITO was also examined in this work.

The optimal dilution of HRP-labeled anti-CEA was established by using different dilutions to react with the analyte CEA in solution and the immobilized CEA on the immunosensor. Figure 5a shows the experimental results. Because the current response tended to a maximum value at a dilution of 1:2, 1:2 dilution of HRP-labeled anti-CEA was chosen as the working solution throughout the experiment. The formation of an immunoconjugate on the CEA-modified ITO electrode surface
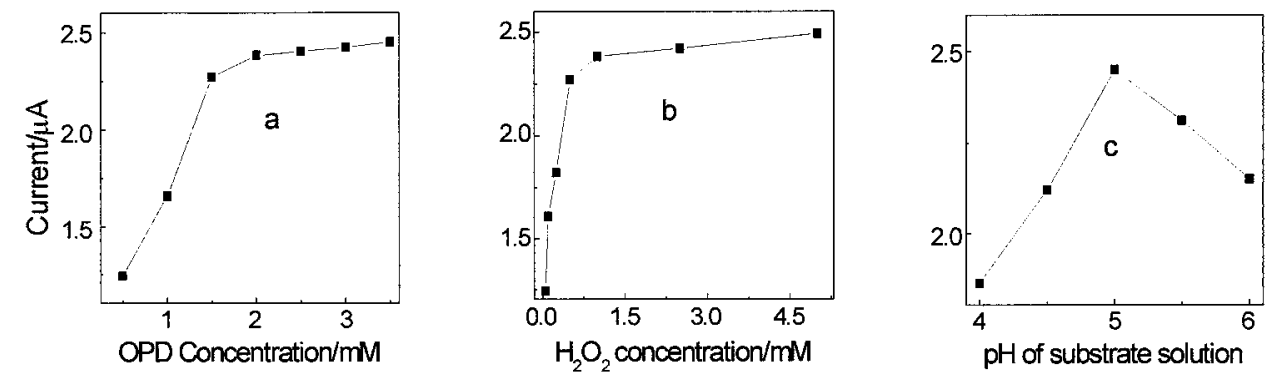

Fig. 4 Effects of (a) concentration of $\mathrm{OPD}$, (b) concentration of $\mathrm{H}_{2} \mathrm{O}_{2}$ and (c) pH of the substrate solution on the DPV peak current. 

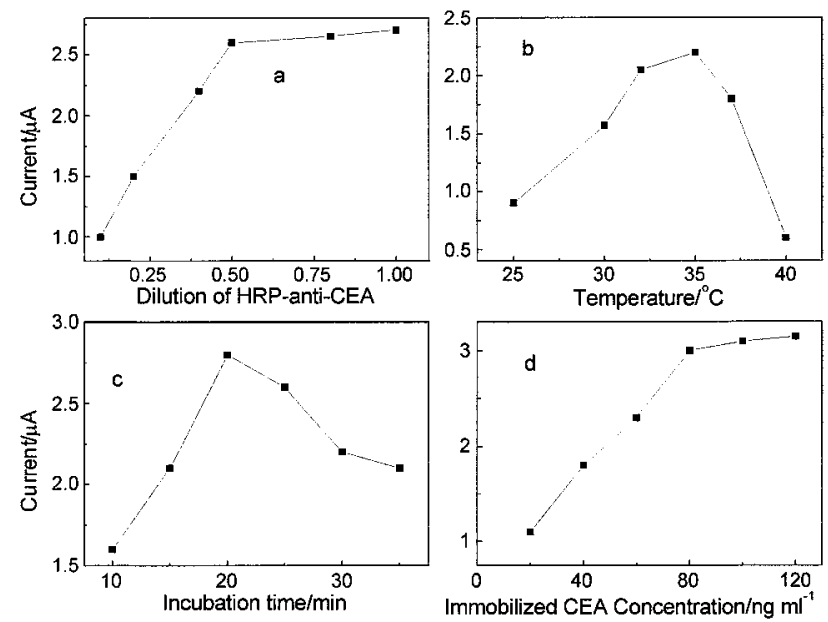

Fig. 5 Effects of (a) dilution of HRP-anti-CEA, (b) incubation temperature, (c) incubation time and (d) immobilized CEA concentration on the DPV peak current of HRP-anti-CEA/CEA/Au-chitosan/ITO in pH 5.0 PBS containing $2.0 \mathrm{mM}$ OPD and $0.5 \mathrm{mM} \mathrm{H}_{2} \mathrm{O}_{2}$.

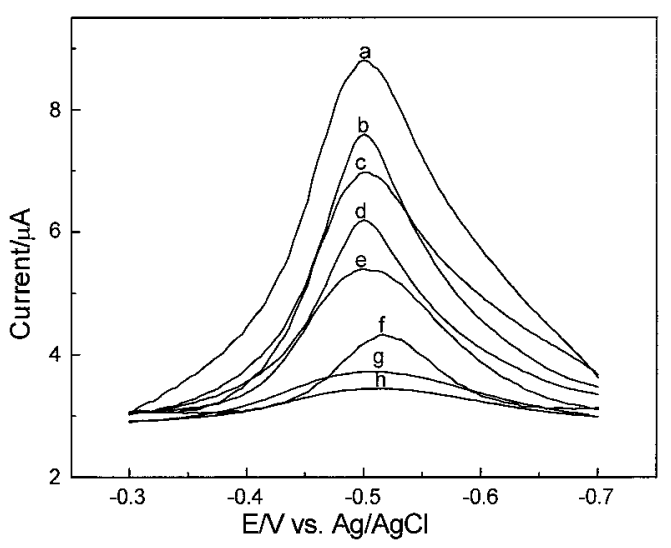

Fig. 6 DPV peak current of HRP-anti-CEA/CEA/Au-chitosan/ITO in $\mathrm{pH}$ 5.0 PBS containing $2.0 \mathrm{mM}$ OPD and $0.5 \mathrm{mM} \mathrm{H}_{2} \mathrm{O}_{2}$ obtained by incubating immunosensor in incubation solutions containing $10 \mu \mathrm{l}$ HRP-anti-CEA and (a) 0, (b) 2.0, (c) 4.0, (d) 8.0, (e) 10.0, (f) 15.0, (g) 17.5 and (h) $20.0 \mathrm{ng} / \mathrm{ml} \mathrm{CEA}$ at $35^{\circ} \mathrm{C}$ for $20 \mathrm{~min}$.

depends on the time and temperature of incubation. A sample containing $15 \mathrm{ng} / \mathrm{ml} \mathrm{CEA}$ was incubated with HRP-labeled antiCEA, and the immunosensor at different temperatures from 25 to $40^{\circ} \mathrm{C}$ was used to select the optimal incubation temperature. As shown in Fig. 5b, the DPV peak current reached the maximum response at $35^{\circ} \mathrm{C}$. At this temperature, the effect of incubation time on the immunoassay was assessed by adding a $10 \mu \mathrm{l} 1: 2$ dilution of HRP-labeled anti-CEA on an antigenmodified ITO electrode surface. The result is shown in Fig. 5c. The current response increased with increasing incubation time, and reached the maximum value at $20 \mathrm{~min}$. When the incubation time was longer than $20 \mathrm{~min}$, the current response changed slightly, indicating that almost the total amount of HRP-labeled CEA antibodies bound to the immobilized CEA antigens. Therefore, an incubation temperature of $35^{\circ} \mathrm{C}$ and an incubation time of $20 \mathrm{~min}$ were chosen as the optimal incubation conditions for the immunoassay. The immunoassay partially depended on the binding of HRP-labeled CEA antibodies to the immunosensor, which was related to the

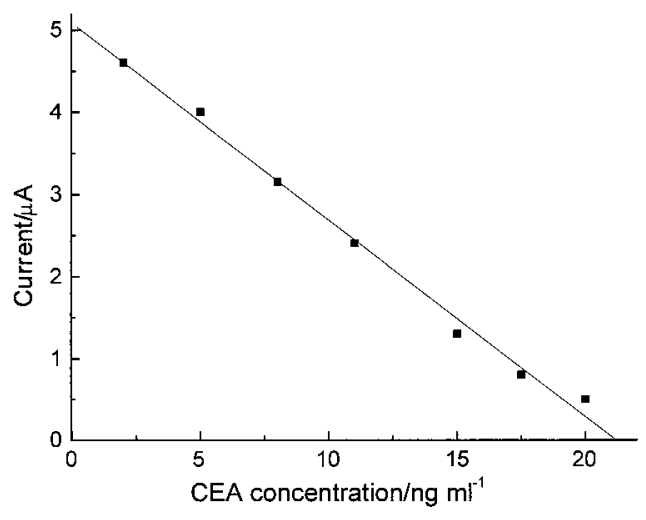

Fig. 7 Calibration linear curve for the competitive immunoassay of CEA.

immobilization and stability of CEA on the Au-chitosan/ITO. An optimal volume of $10 \mu \mathrm{l}$ for $80 \mathrm{ng} / \mathrm{ml}$ CEA antigen was selected in this work, as shown in Fig. 5d.

\section{Calibration curve of the CEA immunosensor}

Under the optimal conditions, the calibration curve for the determination of CEA was obtained by using a CEA/Auchitosan modified ITO electrode as the immunosensor. The DPV peak currents obtained with the competitive immunoassay for CEA are shown in Fig. 6. With increasing concentration of the analyte CEA antigen, the amount of immunoconjugate formed by the antigen and labeled antibody in the solution increased, while the immunoconjugate formed by immobilized CEA and labeled antibody on the CEA/Au-chitosan/ITO electrode surface decreased. Thus, the DPV peak current response of the immunosensor decreased with increasing concentration of the analyte CEA. A plot of the current response $v s$. the CEA concentration shows a linear decrease in the concentration range of CEA from 2.0 to $20 \mathrm{ng} / \mathrm{ml}$ with a correlation coefficient of 0.997 (Fig. 7). The detection limit, taken as the concentration equivalent to a $10 \%$ decrease in the signal, ${ }^{16,36,49,50}$ was calculated to be $1.0 \mathrm{ng} / \mathrm{ml}$. When the CEA concentration was higher than $20 \mathrm{ng} / \mathrm{ml}$, the detection needed an appropriate dilution of the sample.

\section{Accuracy and clinical application}

The accuracy of CEA detection was examined by comparing the analytical results of five serum samples with this method and IRMA. The mean CEA concentrations determined with IRMA, were 5.1, 10.4, 16.3, 16.4 and $13.9 \mathrm{ng} / \mathrm{ml}$, respectively, while this method gave the values of $5.4,9.8,15.5,17.8$ and $12.9 \mathrm{ng} / \mathrm{ml}$, respectively. The relative deviations between the two methods were $5.89,-5.77,4.90,8.54$ and $-7.19 \%$. Thus, the two methods were in acceptable agreement.

\section{Stability and reproducibility of the CEA immunosensor}

The stability of the CEA immunosensor was assessed with intra- and inter-assay variation coefficients (CVs). The intraassay $\mathrm{CV}$ was the difference among three determinations of one sample on the same CEA/Au-chitosan modified ITO electrode after the immunosensor was regenerated. The inter-assay CV was the difference among the measurements of one sample on three different CEA/Au-chitosan modified ITO electrodes. The intra- and inter-assay CVs obtained at a CEA concentration of $8.0 \mathrm{ng} / \mathrm{ml}$ were 9.5 and $4.5 \%$, respectively. Obviously, the preparation of the immunosensor possessed acceptable 
reproducibility. After the immunosensor was stored at $4{ }^{\circ} \mathrm{C}$, it retained $90 \%$ of its initial current after a storage period of 2 weeks. These results demonstrated that the immunosensor possessed good stability and acceptable reproducibility, and could be prepared in batch.

\section{Conclusions}

A disposable electrochemical immunosensor for CEA was prepared by immobilizing CEA antigen on a Au-chitosan membrane modified ITO electrode surface. A competitive immunoassay format resulted in the binding of HRP-anti-CEA antibody to the immobilized CEA antigen. Under catalysis of the formed immobilized HRP-labeled antibody, the amperometric response of the enzymatic reaction product was used for determining the serum CEA level. This prepared CEA immunosenor is more economic due to the low-cost ITO film electrode obtained from industrial mass production compared to the method usng a glassy-carbon electrode. ${ }^{37}$ It possesses acceptable detection and storage stability for fabrication in batch. This low-cost and flexible electrochemical immunosensor enables a more simple and economic immunoassay for CEA and other clinically important antigens.

\section{Acknowledgements}

This work was supported by the Doctoral Foundation of QUST (0022141), the Program for New Century Excellent Talents in Universities (No. NCET-04-0649) and the National Natural Science Foundation of China (No. 20475030).

\section{References}

1. M. H. Schlageter, J. Larghero, and B. Cassinat, Clin. Chem., 1998, 44, 1995.

2. P. Gold and S. Q. Freedman, J. Exp. Med., 1965, 121, 439.

3. M. J. Duffy, A. van Dalen, and C. Haglund, Eur. J. Cancer, 2003, 39, 718.

4. J. P. Kleisbauer, O. Castelnau, and P. Thomas, Lung Cancer, 1996, 15, 148.

5. L. Hernández, A. Espasa, and C. Fernández, Lung Cancer, 2002, 36, 83

6. M. J. A. Engelen, H. W. A. de Bruijn, and H. Hollema, Gynecol. Oncol., 2000, 78, 16.

7. B. Sahin, V. Aydak, and S. Paydas, Eur. J. Cancer, 1996, 32, s24.

8. K. Bremer, S. Micus, and G. Bremer, Eur. J. Cancer, 1995, $31, \mathrm{~s} 262$.

9. B. Jezeršek, J. Cervek, and Z. Rudolf, Cancer Lett., 1996, 110, 137.

10. K. Kudoh, Y. Kikuchi, and T. Kita, Gynecol. Obstet. Invest., 1999, 47, 52.

11. M. Tachibana, Y. Takemoto, and Y. Nakashima, J. Am. College Surg., 1998, 187, 64.

12. S. Y. Kau, Y. M. Shyr, and C. H. Su, J. Am. College Surg., 1999, $188,415$.

13. P. Lechner, P. Lind, and D. M. Goldenberg, J. Am. College Surg., 2000, 191, 511.

14. S. Takamori, J. Yoshimoto, and K. Kojima, Hepatol. Res., 1997, $8,81$.

15. Z. Dai, J. Chen, F. Yan, and H.-X. Ju, Cancer Detection and Prevention, 2005, 29, 233.
16. J.-H. Lin, F. Yan, and H.-X. Ju, Clin. Chim. Acta, 2004, $341,109$.

17. J. C. Forest, J. Masse, and A. A. Lane, Clin. Biochem., 1998, 31,81 .

18. F. Rainer, A. Steffen, and R. Barbara, Luminescence, 2000, 15, 283.

19. V. M. Mekler and S. M. Bystryak, Anal. Chim. Acta, 1992, 264, 359.

20. J. Spona, A. C. Kessler, and R. Deeg, J. Clin. Chem., 1987, $25,53$.

21. K. Matsumoto, J.-L. Yuan, and G.-L. Wang, Anal. Biochem., 1999, 276, 81.

22. M. Haga, S. Hoshino, and H. Okada, Chem. Pharm. Bull., 1990, 38, 252.

23. M. Cioffi, M. T. Vietri, and P. Gazzerro, Lung Cancer, 2001, 33, 163.

24. J. Zhang, K. H. Au, Z. Q. Zhu, and S. O'Shea, Optical Materials, 2004, 26, 47.

25. J. D. Zhang, M. Kambayashi, and M. Oyama, Electroanalysis, 2005, 17, 408.

26. K. Hayashi, Y. Iwasaki, and T. Horiuchi, Anal. Chem., 2005, 77, 5236.

27. X. H. Sun and K. D. Gillis, Anal. Chem., 2006, 78, 2521.

28. A. N. Shipway, E. Katz, and I. Willner, Chem. Phys. Chem., 2000, 1, 18.

29. M. Tominaga, T. Kumagai, and S. Takita, Chem. Lett., 1993, $1,1771$.

30. M. Martinez, J. Herrero, and M. T. Gutierrez, Electrochim. Acta, 1992, 37, 2565.

31. P. M. Armistead and H. H. Thorp, Anal. Chem., 2001, 73, 558.

32. W. Zhan, J. Alvarez, and R. M. Crooks, J. Am. Chem. Soc., 2002, 124, 13265.

33. H. Qiu, J. Yan, and X. Sun, Anal. Chem., 2003, 75, 5435.

34. B. Corry, J. Uilk, and C. Crawley, Anal. Chim. Acta, 2003, 496, 103.

35. A. N. Asanov, W. W. Wilson, and P. B. Oldham, Anal. Chem., 1998, 70, 1156.

36. J.-H. Lin, F. Yan, X.-Y. Hu, and H.-X. Ju, J. Immunol. Methods, 2004, 291, 165.

37. J. Chen, F. Yan, D. Du, J. Wu, and H.-X. Ju, Electroanalysis, 2006, 18, 670.

38. H. Yu, F. Yan, Z. Dai, and H.-X. Ju, Anal. Biochem., 2004 331,98

39. O. D. Velev and E. W. Kaler, Langmuir, 1999, 15, 3693.

40. M. Dequarie, C. Degrand, and B. Limoges, Anal. Chem., 2000, $72,5521$.

41. L.-N. Wu, J. Chen, D. Du, and H.-X. Ju, Electrochim. Acta, 2006, 51, 1208.

42. W. Cheng, S.-J. Dong, and E.-K. Wang, Langmuir, 2002, $18,9947$.

43. A. Doron, E. Katz, and I. Willner, Langmuir, 1995, 11, 1313.

44. H.-Z. Huang and X.-R. Yang, Colloids Surf., A, 2003, 226, 77.

45. C.-X. Lei, F.-C. Gong, G.-L. Shen, and R.-Q. Yu, Sens. Actuators, B, 2003, 96, 582.

46. Y. Xiao, H.-X. Ju, and H.-Y. Chen, Anal. Chim. Acta, 1999, 391, 73 .

47. J.-H. Lin, W. Qu, and S.-S. Zhang, Anal. Biochem., 2007, 360, 288.

48. B. Porstmann and T. Porstmann, "Non-isotopic Immunoassay", 4th ed., 1988, Plenum Press, New York.

49. J. Gascón, G. Durand, and D. Barcelo, Environ. Sci. Technol., 1995, 29, 1551.

50. R. W. Keay and C. J. McNeil, Biosens. Bioelectron., 1998 13,963 . 